\title{
Importance of Nutritional Evaluation in Transplant Patients Awaiting Lung Transplantation
}

\section{Sevan Çetin Özbek ${ }^{1 *}$ and Fatma Perim Türker ${ }^{2}$}

${ }^{1}$ Department of Nutrition and Dietetics, Faculty of Health Sciences Yüksek Ihtisas University, Ankara, Turkey

${ }^{2}$ Department of Nutrition and Dietetics, Faculty of Health Sciences, Baskent University, Ankara, Turkey

*Corresponding Author: Sevan Çetin Özbek, Assistant Professor, Department of Nutrition and Dietetics, Faculty of Health Sciences Yüksek Ihtisas University, Ankara, Turkey.
Received: June 30, 2021

Published: July 20, 2021

(C) All rights are reserved by Sevan Çetin

Özbek and Fatma Perim Türker.

\begin{abstract}
Nutritional status of lung transplant candidates is important both before and after transplant. Early assessment of nutritional status is necessary to identify individuals with malnutrition/at risk of malnutrition and to prevent complications, manage the catabolic process and provide optimal nutrition. In this article, it is aimed to draw attention to the importance of nutritional evaluation before lung transplantation. By reason of today, there is still a need to increase malnutrition awareness, prevent malnutrition and spread interdisciplinary work all over the world. Malnutrition and obesity, considered, a malnutrition, have negative consequences for the patient, health professionals and the economy. These are encountered with deterioration of organ systems, increase in hospital stay-cost-re-admission, in morbidity and mortality. For this reason, determining the nutritional problems of the patients from the first admission to the hospital is the key point in predicting the problem from the beginning and producing a solution accordingly. In particular, the nutritional status of lung transplant candidates affects short and long-term factors such as post-transplant complications, length of hospital stay and mortality, post-discharge care and re-admission to the hospital.
\end{abstract}

Keywords: Lung Transplantation; Nutritional Status; Evaluation of Nutritional Status

\section{Abbreviations}

BMI: Body Mass Index; PNI: Prognostic Risk İndex

\section{Introduction}

The respiratory system in the human body consists of the nose, oropharynx, larynx, trachea, bronchi, bronchioles and lungs. The lungs are anatomically connected to the trachea through the right and left bronchi; taking oxygen into the body and removing carbon dioxide; It is an organ responsible for gas exchange [1]. The lungs are anatomically located in the thoracic cavity and have 3 lobes on the right and two lobes on the left [2]. The openness of the lungs to environmental exposures is in parallel with the exposure to da- mage. Death rates from lung diseases in the world are approximately 3 million people with chronic obstructive pulmonary disease, 250,000 to 500,000 people with respiratory tract infections, 1.4 million people with tuberculosis and 1.6 million people with lung cancer [3]. Lung transplantation has become increasingly common in recent years and all over the world [4]. At the same time the last stage is a treatment model that increases the survival and quality of life for lung patients $[4,5]$. According to the reports of lung transplant centers, the data of June 2018 indicate that 69,200 lung transplants have been performed until this date and the number of centers performing more than 50 transplants per year has increased from 18 to 20 [6]. A total of 34024 transplants were performed in Europe in 2017, including heart, kidney and liver transplants, of 
which 2013 were lung transplants [7]. In our country, the first lung transplantation was performed in 2004 and the number of licensed centers performing lung transplantation has increased gradually since then. The number of lung transplants performed in these centers until 2017 is 168, and it is stated that an average of 30 lung transplants are performed annually in our country [8].

The multidisciplinary 'Transplant Teams' consisting of a transplant surgeon and doctor, transplant coordinator, dietitian, psychiatrist/psychologist, social worker and pharmacist, have a long and difficult journey with the patient [9]. The task of this team is to evaluate the suitability of the transplant candidate, to prepare the patient for the transplant process, to ensure and maintain the quality of life, and to work towards increasing survival after the transplant [10].

In the technical report published by the experts of the World Health Organization and the Food and Agriculture Organization in 2002 , the importance of nutrition in the fight against non-communicable diseases were emphasized [11]. On the other hand, maintaining nutrition and medical nutrition therapy are an integral part of the transplantation process, which is a long journey for healthcare professionals and patients [12].

The International Heart and Lung Transplantation Society has specified the general criteria required to perform transplantation in end-stage lung patients. Among these criteria, progressive or severe malnutrition and class I obesity (Body Mass Index-BMI 30.0 - $34.9 \mathrm{~kg} / \mathrm{m}^{2}$ : especially central obesity) are considered relative contraindications, and class II and III obesity (BMI $\left.\geq 35 \mathrm{~kg} / \mathrm{m}^{2}\right)$ are considered absolute contraindications [16].

This article emphasizes the importance of evaluating nutritional status in lung transplant candidates.

\section{Lung transplantation and malnutrition}

Malnutrition is a common condition at admission to hospital, during hospitalization and after surgery [17]. The majority of lung transplant candidates suffering from malnutrition $[10,18]$.

While the European Society for Clinical Nutrition and Metabolism Association defines malnutrition as "decreased physical and mental functions and worsening of the clinical outcome of the disease as a result of deterioration in body composition (decrease in lean mass) and body cell mass, caused by inadequate intake or irregular nutrition" [19], the American Society The Association for Parenteral and Enteral Nutrition and the Academy of Nutrition and Dietetics recommend 2 or more of the characteristics of low energy consumption, weight loss, subcutaneous fat loss, loss of muscle mass, fluid accumulation (leucalized or general), and functional capacity (determined by measurements) in adult malnutrition accepted the presence of excess as adult malnutrition [20].

The negative effects of malnutrition are multi-faceted. It can affect all organ systems and quality of life in the patient, such as deterioration in bowel integrity and immunity, increase in infection rates, delay in wound healing, hypothermia, deterioration in renal functions, decrease in cardiac output, increase in psychological problems such as apathy/depression, loss of muscle mass, deterioration in liver functions [21].

On the other hand, it also has economic and political repercussions with the increase in the length of hospital stay and re-admissions, mortality and health expenditures [20,21]. Identification of patients with malnutrition and/or malnutrition risk is the basis of the solution step in preventing the risk before malnutrition occurs or in early intervention in malnutrition. For nutritional physiology is complex, complicate and reveals individual results depending on the nutritional status of the person [22].

The presence of malnutrition is a relative contraindication for lung transplant candidates [23] and cachexia may be observed in advanced-stage patients (chronic obstructive pulmonary disease, idiopathic cystic fibrosis) due to the presence of inflammation [24]. However, while the threshold value for obese individuals was determined as BMI $>35 \mathrm{~kg} / \mathrm{m}^{2}$. The threshold value in body mass index for lung transplant candidates with malnutrition is not clear [25]. In end-stage lung disease, malnutrition occurs due to decreased food intake, changes in metabolism, inflammation, malabsorption, and infections [10]. The presence of malnutrition in a patient who will have a lung transplant may also affect post-transplant morbidity and mortality $[10,18]$.

In a retrospective study conducted at the University of Strasbourg, they stood out to nutritional deficiencies among lung transplant candidates. In the study in which the BMIs (1 month, 3 months, 6 months and 1 year) and nutritional status of the patients were followed up, a decrease was found in BMI during the 
lung transplantation (pre-transplant hospitalization, ICU process); It has been stated that it takes about 1 year to replace the lost weight and reach the pre-transplant BMI level. In addition, it was stated that keeping the BMI at the level of $22.8 \mathrm{~kg} / \mathrm{m}^{2}$ before the transplant can prevent the risk of BMI falling below $21 \mathrm{~kg} / \mathrm{m}^{2}$ after the transplant [25]. In the study examining the nutritional profile of lung transplant candidates, it was found that the groups with low BMI, triceps skinfold thickness and upper-middle arm muscle circumference, respectively, were in cystic fibrosis (40\%-64.7\%$76.5 \%)$ and bronchiectasis (32\%-52.6\%-73.7\%) patients stated [26].

Evaluation of nutritional status is a part of the nutritional care process and is a comprehensive method used to identify nutritional problems (anthropometric methods, nutritional history, laboratory findings, medical history, physical nutrition examination) in patients. At the same time, the evaluation of nutritional status lays the foundations for the clinical decision regarding the next nutrition to be applied to the patient and establishes the future procedure [27].

In a study evaluating the prognostic risk index (PNI) score and nutritional status of lung transplant candidates in the preoperative period, it was shown that individuals with a low PNI score (PNI $<41.5$ ) have high mortality and complication rates. The investigators also evaluated the incidence of post-operative complications with the preoperative PNI score. According to the Claviene Dindo surgical complication classification, there were significantly higher complications in the PNI-low group than in the PNI-high group (71.4\% vs. 23.2\%; $\mathrm{P}<0.001$ ) [15].

In a research based on the hypothesis that nutritional status deteriorates before and after transplantation and decrease in body mass is a risk factor for mortality, it was shown that nutritional status was impaired in $72 \%$ of cases, and reductions in lean body mass occurred in $17 \%$ of cases without weight loss [28]. In a retrospective study examining the effect of nutritional status on mortality in single and bilateral lung transplant patients, it was stated that a $\mathrm{BMI}>27.5 \mathrm{~kg} / \mathrm{m}^{2}$ in the pretransplant period was a predictor for mortality in the post-transplant period. On the other hand, in the study in which prealbumin, which is an indicator of nutritional status, was evaluated, the risk of death was 3 times higher in patients with prealbumin level $\leq 18 \mathrm{~g} / \mathrm{dL}$ compared to patients with $>18 \mathrm{~g} /$ $\mathrm{dL}$ (HR 3.01, 95\% CI: 1.21 to 7.51; $\mathrm{P}=0.018$ ) has been shown [29].
Obesity is a factor that affects surgery and overall health due to its comorbidities and complications worldwide [30]. On the other hand, today it is accepted in the malnutrition class due to the negative results it creates in the organism [31].

While obesity is associated with results such as changes in the pharmacokinetics of drugs, adaptation to drug doses, and limitation of movement, especially in maintaining the airway in intensive care patients [32,33], it is also associated with negative outcomes that may occur with comorbidities such as Type 2 Diabetes Mellitus, hypertension and asthma [32]. Obesity has a negative effect on the lungs with increased amount of adipose tissue in the thorax, abdomen and internal organs (visceral). Therefore, this makes breathing difficult and reduces functional residual capacity [34]. The American Society of Transplant Physicians, the American Thoracic Society, the European Respiratory Society, and the International Society for Heart and Lung Transplantation, in the guidelines published in 1998, stated that for transplantation, weight gain or loss in individuals with $<70 \%$ and $>130 \%$ of their ideal body weight. suggested that they take action in this direction [35].

In a study examining the relationship between preoperative nutritional status and mortality in the first 3 months (90 days) after lung transplantation, low BMI $<17 \mathrm{~kg} / \mathrm{m}^{2}$ or $>25 \mathrm{~kg} / \mathrm{m}^{2}$ was shown as a factor increasing the mortality risk. It has been reported that patients with a pre-BMI $>27 \mathrm{~kg} / \mathrm{m}^{2}$ have a higher BMI than the reference group (BMI $20-25 \mathrm{~kg} / \mathrm{m}^{2}$ ) [36]. In a study examining the effect of body mass index in lung transplantation in 546 patients, it was shown that the mortality rate is high in overweight and obese individuals [37].

\section{Conclusion}

Nutritional assessment is essential for transplant patients. However, it is even more important to create an individual nutrition care plan according to the weight loss/gain of the patients during the transplantation process. Evaluation of nutritional status in predicting nutrition-related problems is a method that facilitates risk and crisis management in the transplant process. Increasing the number of 'Transplant Teams' aiming the maximum benefit to the patient, such as 'Nutrition Teams' where multidisciplinary work is adopted; in this regard, it will provide a great advantage to both patients and healthcare professionals. 


\section{Conflict of Interest}

The authors declare that there is no conflict of interest.

\section{Bibliography}

1. Haddad Moshe and Sharma Sandeep. "Physiology". Lung (2021).

2. Kılıç Cenk. "Akciğerlerin Anatomisi". Journal of Clinical and Analytical Medicine (2011):1-3.

3. Forum of International Respiratory Societies. "The Global Impact of Respiratory Disease-Second Edition” (2021).

4. Girgis Reda E and Khaghani Asghar. "A global perspective of lung transplantation: Part 1 - Recipient selection and choice of procedure". Global Cardiology Science and Practice (2016): 5 .

5. Jomphe Valerie., et al. "Nutritional Requirements of Lung Transplant Recipients: Challenges and Considerations". Nutrients 10 (2018): 790.

6. Chambers Daniel C., et al. "The International Thoracic Organ Transplant Registry of the International Society for Heart and Lung Transplantation: Thirty-sixth adult lung and heart-lung transplantation Report-2019; Focus theme: Donor and recipient size match". The Journal of Heart and Lung Transplantation 38 (2019): 1042-1055.

7. European Kidney Health Alliance. "Organ Donation and Transplantation in the EU" (2021).

8. Turkish Thoracic Society. "Lung Transplantation in Our Country" (2021).

9. American Transplant Foundation. "The Transplant Team" (2021).

10. Tynan Cameo and Hasse Jeanette M. "Current nutrition practices in adult lung transplantation". Nutrition in Clinical Practice 19 (2004): 587-596.

11. WHO Tecnical Report Series. "Diet, nutrition and the prevention of chronic diseases" (2021).
12. Skipper Annalynn. "Dietititan's Handbook of Enteral and Parenteral Nutrition". Trans. Besler, Tanju H, İnanç, Neriman. İstanbul: Nobel Tıp Kitapevi (2014).

13. Weimann Arved., et al. "ESPEN guidelines on enteral nutrition: Surgery including organ transplantation". Clinical Nutrition 25 (2006): 224-244.

14. Shah Pali and Orens Jonathan B. "Impact of nutritional state on lung transplant outcomes: The weight of the evidence". The Journal of Heart and Lung Transplantation 32 (2013): 755756.

15. Kim Chi Y., et al. "Usefulness of the preoperative prognostic nutritional index score as a predictor of the outcomes of lung transplantation: A single-institution experience". Clinical $\mathrm{Nu}$ trition 38 (2019): 2423-2429.

16. Weil David., et al. "A consensus document for the selection of lung transplant candidates: 2014-An update from the Pulmonary Transplantation Council of the International Society for Heart and Lung Transplantation". The Journal of Heart and Lung Transplantation 34 (2015): 1-15.

17. Barker Lisa A., et al. "Hospital Malnutrition: Prevalence, identification and impact on patients and the healthcare system". International Journal of Environmental Research and Public Health 8 (2011): 514-527.

18. Chohan Karan., et al. "Evaluation of malnutrition risk in lung transplant candidates using the nutritional risk index". Transplantation Direct 6 (2020): e574.

19. Cederholm Tommy., et al. "ESPEN guidelines on definitions and terminology of clinical nutrition". Clinical Nutrition 36 (2017): 49-64.

20. White Jane V., et al. "Consensus statement: Academy of Nutrition and Dietetics and American Society for Parenteral and Enteral Nutrition: characteristics recommended for the identification and documentation of adult malnutrition (undernutrition)". Journal of Parenteral and Enteral Nutrition 36 (2012): 275-283.

21. Saunders John., et al. "Malnutrition and undernutrition". Medicine 39 (2011): 45-50. 
22. Reber Emilie., et al. "Nutritional risk screening and assessment". Journal of Clinical Medicine 8 (2019): 1065.

23. Weill David. "Lung transplantation: indications and contraindications". Journal of Thoracic Disease 10 (2018): 4574-4587.

24. Sanders Karin J., et al. "Cachexia in chronic obstructive pulmonary disease: new insights and therapeutic perspective". Journal of Cachexia, Sarcopenia and Muscle 7 (2016): 5-22.

25. Boura Sophie., et al. "Optimization of nutritional management of patients awaiting lung transplant at the Strasbourg University Hospitals". Clinical Nutrition Experimental 27 (2019): 9-20.

26. Souza Sabrina MP de., et al. "Nutritional profile of lung transplant candidates". Jornal Brasileiro de Pneumologia 35 (2009): 242-247.

27. Mueller Charles., et al. "A.S.P.E.N. clinical guidelines: Nutrition screening, assessment, and intervention in adults". Journal of Parenteral and Enteral Nutrition 35 (2011): 16-24.

28. Schwebel David C., et al. " Prevalence and consequences of nutritional depletion in lung transplant candidates". European Respiratory Journal 16 (2000): 1050-1055.

29. González-Castro Alejandro., et al. "Influence of Nutritional Status in Lung Transplant Recipients". Transplantation Proceedings 38 (2006): 2539-2540.

30. DiCecco Sara R and Francisco-Ziller Nickie. "Obesity and Organ Transplantation: Successes, failures, and opportunities". Nutrition in Clinical Practice 29 (2014): 171-191.

31. Barazzoni Rocco and Cappellari Gianluca G. "Double burden of malnutrition in persons with obesity". Reviews in Endocrine and Metabolic Disorders 21 (2020): 307-313.

32. Malhotra Atul and Hillman David. "Obesity and the lung: 3. Obesity, respiration and intensive care". Thorax 63 (2008): 925-931.

33. Schetz Miet., et al. "Obesity in the critically ill: a narrative review”. Intensive Care Medicine 45 (2019): 757-769.

34. Salome Cheryl M., et al. "Physiology of obesity and effects on lung function". Journal of Applied Physiology 108 (2010): 206211.
35. American Thoracic Society. "International guidelines for the selection of lung transplant candidates" (2021).

36. Madill Janet., et al. "Nutritional Assessment of the Lung transplant patient: Body mass index as a predictor of 90-day mortality following transplantation". The Journal of Heart and Lung Transplantation 20 (2001): 288-296.

37. Ruttens David., et al. "Body mass index in lung transplant candidates: a contra-indication to transplant or not? Transplantation Proceedings 46 (2014): 1506-1510.

\section{Volume 4 Issue 8 August 2021}

(C) All rights are reserved by Sevan Çetin Özbek and Fatma Perim Türker. 